\title{
The polyene antifungals, amphotericin $B$ and nystatin, cause cell death in Saccharomyces cerevisiae by a distinct mechanism to amphibian-derived antimicrobial peptides
}

\author{
George Serhan, Colin M Stack, Gabriel G Perrone and Charles Oliver Morton ${ }^{*}$
}

\begin{abstract}
Background: There is a pressing need to identify novel antifungal drug targets to aid in the therapy of life-threatening mycoses and overcome increasing drug resistance. Identifying specific mechanisms of action of membrane-interacting antimicrobial drugs on the model fungus Saccharomyces cerevisiae is one avenue towards addressing this issue. The $S$. cerevisiae deletion mutants $\Delta i z h 2, \Delta i z h 3, \Delta$ aif1 and $\Delta s t m 1$ were demonstrated to be resistant to amphibian-derived antimicrobial peptides (AMPs). The purpose of this study was to examine whether AMPs and polyene antifungals have a similar mode of action; this was done by comparing the relative tolerance of the mutants listed above to both classes of antifungal.

Findings: In support of previous findings on solid media it was shown that $\Delta i z h 2$ and $\Delta i z h 3$ mutants had increased resistance to both amphotericin $B\left(1-2 \mu \mathrm{g} \mathrm{ml}^{-1}\right)$ and nystatin $\left(2.5-5 \mu \mathrm{g} \mathrm{m}{ }^{-1}\right)$ in liquid culture, after acute exposure. However, $\Delta$ aif1 and $\Delta s t m 1$ had wild-type levels of susceptibility to these polyenes. The generation of reactive oxygen species (ROS) after exposure to amphotericin B was also reduced in $\Delta i z h 2$ and $\triangle i z h 3$. These data indicated that polyene antifungal and AMPs may act via distinct mechanisms of inducing cell death in S. cerevisiae.

Conclusions: Further understanding of the mechanism(s) involved in causing cell death and the roles of IZH2 and IZH3 in drug susceptibility may help to inform improved drug design and treatment of fungal pathogens.
\end{abstract}

Keywords: Amphotericin B, Antifungal resistance, YOLO02C, Antifungals

\section{Findings}

The incidence of invasive fungal diseases is increasing, especially in those individuals undergoing immunosuppressive treatments or those with predisposing conditions [1]. The number of antifungal agents available is limited due to the biological overlap and conserved nature of cellular processes between fungi and mammalian cells. Antifungal drug resistance, particularly to azoles, is also increasing which has created a strong need for the identification of novel antifungal therapies and drug targets [2].

The major targets for antifungal drugs are the cell wall and plasma membrane. The major groups of antifungals in use today are the polyene (amphotericin B and nystatin)

\footnotetext{
* Correspondence: o.morton@uws.edu.au

University of Western Sydney, School of Science and Health, Campbelltown Campus, Narellan Road, Campbelltown, NSW 2560, Australia
}

and azole (e.g. voriconazole) classes which target ergosterol in the fungal plasma membrane and ergosterol synthesis, respectively [3]. In fungi ergosterol serves an analogous function to cholesterol in mammalian cell membranes and forms the basis of the selective toxicity of many antifungals. Azoles target the proteins responsible for ergosterol biosynthesis whereas polyenes interact with ergosterol in the plasma membrane [4]. Resistance to azoles has been shown to result from nucleotide polymorphisms is the cyp51a gene whereas resistance to polyenes is less common due to it targeting an essential structural component of the membrane [5]. Polyenes primarily act by binding membrane ergosterol and forming pores in the plasma membrane which is similar to the proposed mode of action for antimicrobial peptides (AMPs). These are a group of peptides that form an important part of the 
innate immune system of animals and include the human defensins [6].

Detailed analysis of the activity of amphibian-derived AMPs indicated that they induced programmed cell death (PCD) in Saccharomyces cerevisiae [7] which was also observed during exposure of Aspergillus fumigatus to amphotericin B [8]. Formation of pores in membranes is now thought to be a secondary inhibitory mechanism for both of these classes of molecule with primary activity leading to intracellular oxidative damage and interaction with organelles [9]. The purpose of this study was to build on previous research that identified S. cerevisiae deletion mutants that were resistant to amphibian-derived AMPs and to examine if they are also resistant to amphotericin B. This may indicate similarities in the mode of action and induction of PCD between these antifungal agents.

The $S$. cerevisiae deletion mutants $\Delta i z h 2, \Delta i z h 3, \Delta s t m 1$ and $\Delta a i f 1$ were selected from the haploid yeast deletion mutant library (strain BY4742 from Thermo Scientific) based on the resistance of these mutants to amphibianderived antimicrobial peptides [7]. Cultures of these strains and wild-type were grown in YPD broth (SigmaAldrich) at $30^{\circ} \mathrm{C}$ with shaking at $150 \mathrm{rpm}$ overnight.
After overnight growth cultures were adjusted to an $\mathrm{OD}_{600}$ of 0.5 before treatment with $1-2 \mu \mathrm{g} \mathrm{ml}{ }^{-1}$ amphotericin B (Sigma-Aldrich) or 2.5-5 $\mu \mathrm{g} \mathrm{ml}^{-1}$ of nystatin (Sigma-Aldrich). These doses are greater than the minimum inhibitory concentrations of the drugs (0.1-2 $\mu \mathrm{g} \mathrm{ml}^{-1}$ amphotericin B and $2.0 \mu \mathrm{g} \mathrm{ml}^{-1}$ nystatin) to test drug resistance. Drug-treated cultures were incubated for one, two and three hours after which serial dilutions of each culture were prepared and plated onto YPD agar; these were incubated at $30^{\circ} \mathrm{C}$ for two days. The plates were examined for growth and the numbers of colony forming units (CFU) were counted. Three independent experiments were conducted for each treatment.

Production of reactive oxygen species (ROS) was assessed to examine secondary effects of polyenes. Cultures were treated overnight with 0.5 or $1.0 \mu \mathrm{g} \mathrm{m} l^{-1}$ amphotericin B and ROS was detected by a previously described method using 2,7-dichlorofluorescein (Sigma Aldrich, St Louis, MO, USA) [7].

Colony forming units (CFU) data were analysed by repeated measures ANOVA comparing each deletion to the wild-type and Bonferroni's multiple comparison to compare individual treatments. The ROS data was
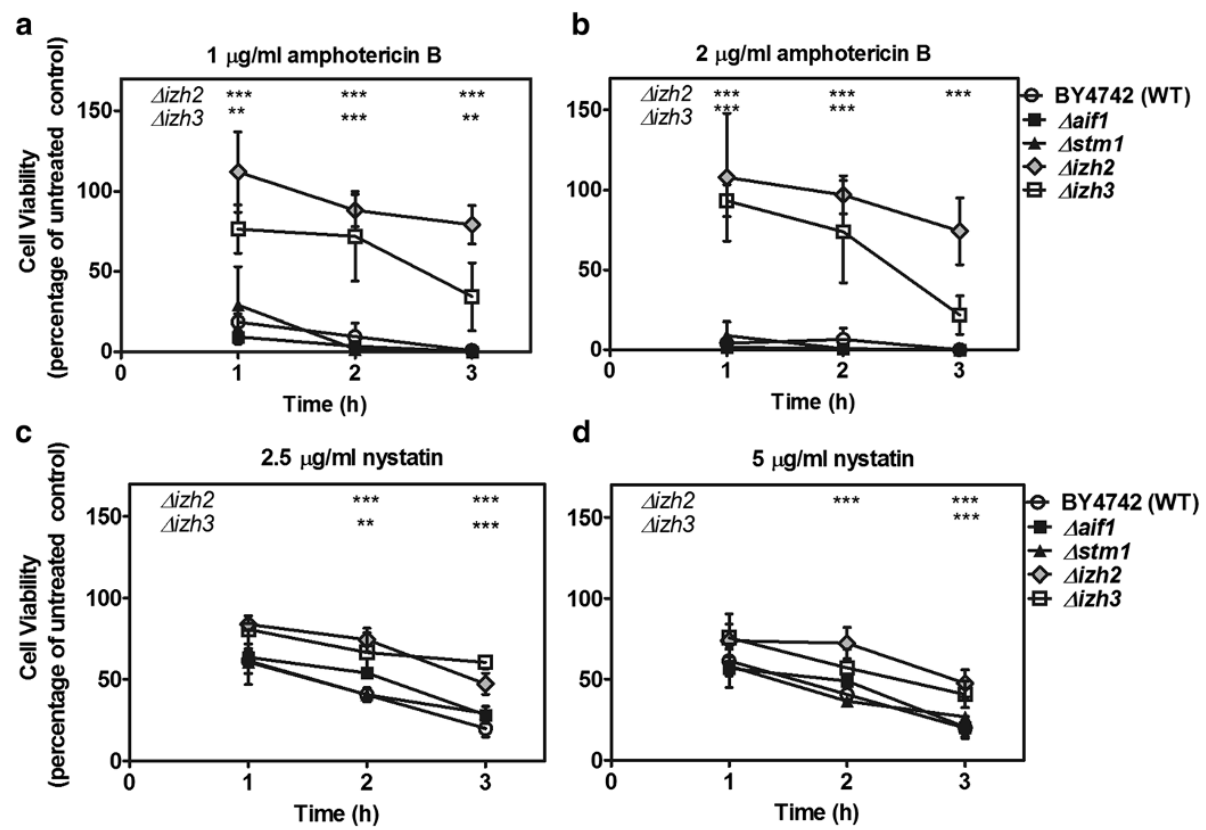

Figure 1 The effects of exposure to polyene antifungals on the wild-type and selected deletion mutants of Saccharomyces cerevisiae. The effect of drug was determined by expressing each treatment as a percentage of control growth to account for variations in starting cell numbers between treatments and experiments. (a-b) Saccharomyces cerevisiae was exposed to two concentrations of amphotericin B; $1.0 \mu \mathrm{g}$ $\mathrm{ml}^{-1}$ and $2.0 \mu \mathrm{g} \mathrm{ml}^{-1}$ for one, two and three hours. The number of viable cells reduced over time for each strain, however $\Delta i z h 2$ and $\Delta i z h 3$ were significantly more resistant than the wild-type and other strains tested at all doses. (c-d) S. cerevisiae was exposed to two concentrations of nystatin; $2.5 \mathrm{\mu g} \mathrm{ml}^{-1}$ and $5.0 \mathrm{\mu g} \mathrm{ml}^{-1}$ for one, two and three hours. The number of viable cells reduced over time for each strain; however $\Delta i z h 2$ and $\Delta i z h 3$ were significantly more resistant than the other strains after two and three hour's incubation. The data in a-d are means and standard deviations of three replicate experiments: these were analysed by repeated measures ANOVA and Bonferroni's multiple comparison test; ${ }^{*} p<0.05$, ${ }^{* *} \mathrm{p}<0.01,{ }^{* * *} \mathrm{p}<0.001$. 
analysed by Mann-Whitney tests comparing treated to untreated cells for each strain.

\section{$\Delta \mathrm{izh} 2$ and $\Delta \mathrm{izh} 3$ exhibit increased resistance to polyene antifungals}

Incubation of wild-type $S$. cerevisiae cells with increasing doses of amphotericin B led to a reduction in viable cells from $80 \%$ viability in the wild type (BY4742) after 1 hour exposure at $1.0 \mu \mathrm{g} \mathrm{ml} \mathrm{m}^{-1}$ to $4 \%$ viability in the wild type after 1 hour exposure to $2.0 \mu \mathrm{g} \mathrm{ml}^{-1}$ of amphotericin B (Figure $1 \mathrm{a}$ and $\mathrm{b}$ ). The absolute values equating to $100 \%$ cell viability were $1 \times 10^{5} \mathrm{CFU} \mathrm{ml} \mathrm{m}^{-1}$ after 1 hour, $1.7 \times$ $10^{5} \mathrm{CFU} \mathrm{ml} \mathrm{m}^{-1}$ after 2 hours and $3.7 \times 10^{5} \mathrm{CFU} \mathrm{ml}^{-1}$ after 3 hours. The wild type, $\Delta a i f 1$ and $\Delta s t m 1$ mutants were highly sensitive to amphotericin B and nystatin with significant reductions in viability over time in a dosedependent manner (Figure $1 \mathrm{a}-\mathrm{d}$ ). The $\Delta i z h 2$ and $\Delta i z h 3$ mutants showed increased resistance to exposure to amphotericin B, which was particularly pronounced at higher drug doses. The most pronounced differences in drug resistance were observed when these deletion mutants were exposed to amphotericin B.

Prolonged exposure to amphotericin B resulted in formation of reaction oxygen species (ROS) in drug-treated $S$. cerevisiae cells, all strains (Figure 2). Amphotericin B treatment significantly increased the number of fluorescent cells induced in BY4742, Daif1 and $\Delta s t m 1$, whereas no significant increase was observed in $\Delta i z h 2$ and $\Delta i z h 3$.

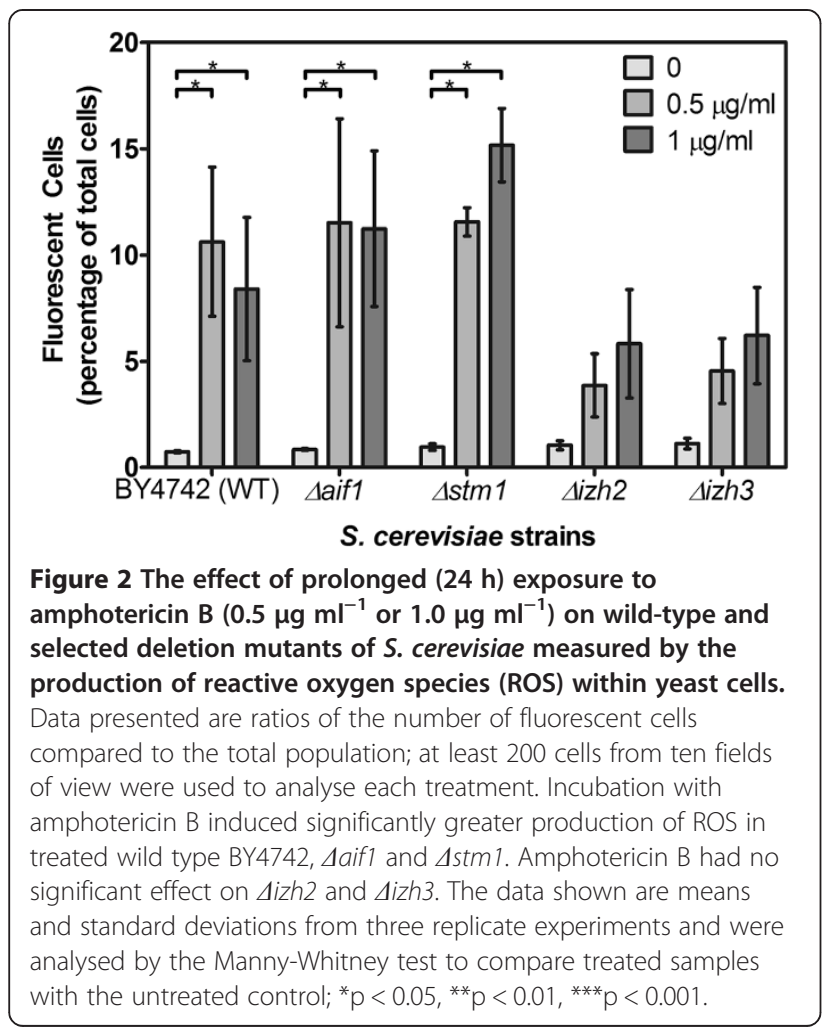

ROS generation may indicate induction of PCD and correlates with the oxidative damage that has been linked to the activity of amphotericin B [10,11]. Similar data were obtained for the reaction of $S$. cerevisiae and $\mathrm{mu}$ tants treated with $5.0 \mu \mathrm{g} \mathrm{ml}^{-1}$ nystatin (data not shown).

\section{Polyene antifungals appear to cause cell death in yeast by a distinct mechanism compared to AMPs}

In this study we demonstrated that deletion of $I Z H 2$ or IZH3 conferred increased resistance to amphotericin B, which was previously reported for amphibian-derived AMPs [7]. AMPs [7] and polyenes (Figure 2) lead to intracellular accumulation of ROS that is associated with oxidative damage and PCD. However, susceptibility of $\triangle$ aif1 and $\Delta s t m 1$ mutants to amphotericin B suggests that a different mechanism may be involved in polyene-mediated cell death. That both of these mechanisms are likely caspase-independent in Aspergillus fumigatus [8] but not in Candida Biofilms [12], which underlines the need to elucidate the variety of mechanisms leading to cell death in microbes in order to exploit these mechanisms as a means of controlling fungal infections.

The major similarity between the two classes of antifungal was the requirement for $I Z H 2$ and $I Z H 3$ for full susceptibility of $S$. cerevisiae to membrane-interacting antifungals. These genes are members of the progestin and adipoQ receptor (PAQR) family, which is a group of membrane embedded receptor molecules [13]. Previous studies had shown that deletion of $I Z H 2$ and $I Z H 3$ led to resistance to polyenes $[14,15]$ and that the plant defence protein osmotin was shown to bind to Izh2p to induce PCD [16]. The effective drug doses in this study were greater than in the previous study which reported polyene resistance in $\Delta i z h 2$ and $\Delta i z h 3$ [15]. This may be attributed to different formulations of the drug and different culture conditions since other studies have tested drug effects using agar media. The proposed mechanism of action of osmotin, a homologue of adiponectin, is to bind specifically to the receptor domain of Izh2p which causes a signal cascade that triggers PCD [17]. It is possible that the polyenes nystatin and amphotericin B interact with izh2p or izh3p to induce PCD in S. cerevisiae; since the PAQR class of proteins is widely distributed in fungi this would provide a target for rational drug design.

The direct activation of a receptor by the selected polyenes may not be the mechanism of action since these antifungals are not structurally similar to osmotin which is a $24 \mathrm{kDa}$ protein that is homologous to adiponectin [16]. The $I Z H$ family of genes has pleiotropic effects on gene expression and their cellular roles cannot yet be functionally assigned to a single biochemical pathway $[18,19]$. Along with being involved in zinc homeostasis this gene family is also associated with sterol metabolism [18,20]. Specifically, it is thought that their function in sterol 
metabolism may influence membrane permeability to alter ion homeostasis [19]. For example deletion of IZH3 exhibits a positive genetic interaction with the genes SCS7 (sphingolipid alpha-hydroxylase) $[21,22]$ and AUR1 (inositol phosphoceramide synthase) [23]. These are involved in the biosynthesis of sphingolipids which are essential components of the cell membrane. Deletion of $\mathrm{IZH} 3$ may therefore alter membrane composition affecting the ability of polyenes to bind membrane ergosterol leading to enhanced resistance.

This study confirms the resistance of $\Delta i z h 3$ and $\Delta i z h 2$ to polyene antifungals and indicates that Izh3p may have functional similarities to Izh2p with regard to membrane ergosterol content. These data also suggest that polyenes and amphibian-derived antimicrobial peptides may induce PCD in S. cerevisiae by distinct mechanisms. A full understanding of mechanisms of cell death induced by these antifungal agents may yield an important advance in the development of new classes of antifungal drugs.

\section{Availability of supporting data}

The data supporting the results of this study are included within this article.

\section{Abbreviations \\ YPD: Yeast extract peptone dextrose; IZH2/3: Implicated in zinc homeostasis; STM1: suppressor of ToM1; AIF1: Apoptosis inducing factor.}

\section{Competing interests}

The authors declare that they have no competing interests that could affect the integrity of this study.

\section{Authors' contributions}

GS performed the experimental work for the study. CS assisted in experimental design and preparation of the manuscript. GP assisted in experimental design, analysis and manuscript preparation. COM designed the study, assisted in data analysis and wrote the manuscript. All authors read and approved the final manuscript.

\section{Acknowledgements}

The authors wish to acknowledge the UWS Honours Programme for enabling George Serhan to undertake this research. The authors would also like to thank Val Barrow for technical support.

Received: 28 February 2014 Accepted: 3 May 2014

Published: 12 May 2014

\section{References}

1. Neofytos D, Horn D, Anaissie E, Steinbach W, Olyaei A, Fishman J, Pfaller M, Chang C, Webster K, Marr K: Epidemiology and outcome of invasive fungal infection in adult hematopoietic stem cell transplant recipients: analysis of Multicenter Prospective Antifungal Therapy (PATH) Alliance registry. Clin Infect Dis 2009, 48:265-273.

2. Cuenca-Estrella M: Antifungal drug resistance mechanisms in pathogenic fungi: from bench to bedside. Clin Microbiol Infect 2013. doi:10.1111/14690691.12495 [Epub ahead of print]

3. Odds FC, Brown AJ, Gow NA: Antifungal agents: mechanisms of action. Trends Microbiol 2003, 11:272-279.

4. Gray KC, Palacios DS, Dailey I, Endo MM, Uno BE, Wilcock BC, Burke MD: Amphotericin primarily kills yeast by simply binding ergosterol. Proc Natl Acad Sci U S A 2012, 109:2234-2239.

5. Mellado E, Garcia-Effron G, Alcazar-Fuoli L, Melchers WJ, Verweij PE, Cuenca-Estrella M, Rodriguez-Tudela JL: A new Aspergillus fumigatus resistance mechanism conferring in vitro cross-resistance to azole antifungals involves a combination of cyp51A alterations. Antimicrob Agents Chemother 2007, 51:1897-1904.

6. Zasloff M: Antimicrobial peptides of multicellular organisms. Nature 2002, 415:389-395.

7. Morton CO, Dos Santos SC, Coote P: An amphibian-derived, cationic, alpha-helical antimicrobial peptide kills yeast by caspase-independent but AIF-dependent programmed cell death. Mol Microbio/ 2007, 65:494-507.

8. Mousavi SA, Robson GD: Oxidative and amphotericin B-mediated cell death in the opportunistic pathogen Aspergillus fumigatus is associated with an apoptotic-like phenotype. Microbiology 2004, 150:1937-1945.

9. Ogita A, Fujita K, Tanaka T: Enhancing effects on vacuole-targeting fungicidal activity of amphotericin B. Front Microbiol 2012, 3:100.

10. Phillips AJ, Sudbery I, Ramsdale M: Apoptosis induced by environmental stresses and amphotericin B in Candida albicans. Proc Natl Acad Sci U S A 2003, 100:14327-14332.

11. Mesa-Arango AC, Scorzoni L, Zaragoza O: It only takes one to do many jobs: Amphotericin B as antifungal and immunomodulatory drug. Front Microbiol 2012, 3:286.

12. Al-Dhaheri RS, Douglas L: Apoptosis in Candida biofilms exposed to amphotericin B. J Med Microbiol 2010, 59:149-157.

13. Smith JL, Kupchak BR, Garitaonandia I, Hoang LK, Maina AS, Regalla LM, Lyons TJ: Heterologous expression of human mPRalpha, mPRbeta and mPRgamma in yeast confirms their ability to function as membrane progesterone receptors. Steroids 2008, 73:1160-1173.

14. Karpichev IV, Small GM: Global regulatory functions of Oaf1p and Pip2p (Oaf2p), transcription factors that regulate genes encoding peroxisomal proteins in Saccharomyces cerevisiae. Mol Cell Biol 1998, 18:6560-6570.

15. Villa NY, Moussatche P, Chamberlin SG, Kumar A, Lyons TJ: Phylogenetic and preliminary phenotypic analysis of yeast PAQR receptors: potential antifungal targets. J Mol Evol 2011, 73:134-152.

16. Narasimhan ML, Coca MA, Jin J, Yamauchi T, Ito Y, Kadowaki T, Kim KK, Pardo JM, Damsz B, Hasegawa PM, Yun DJ, Bressan RA: Osmotin is a homolog of mammalian adiponectin and controls apoptosis in yeast through a homolog of mammalian adiponectin receptor. Mol Cell 2005, 17:171-180.

17. Villa NY, Kupchak BR, Garitaonandia I, Smith JL, Alonso E, Alford C, Cowart LA, Hannun YA, Lyons TJ: Sphingolipids function as downstream effectors of a fungal PAQR. Mol Pharmacol 2009, 75:866-875.

18. Kupchak BR, Villa NY, Kulemina LV, Lyons TJ: Dissecting the regulation of yeast genes by the osmotin receptor. Biochem Biophys Res Commun 2008, 374:210-213.

19. Lyons TJ, Villa NY, Regalla LM, Kupchak BR, Vagstad A, Eide DJ: Metalloregulation of yeast membrane steroid receptor homologs. Proc Natl Acad Sci U S A 2004, 101:5506-5511.

20. Karpichev IV, Cornivelli L, Small GM: Multiple regulatory roles of a novel Saccharomyces cerevisiae protein, encoded by YOL002c, in lipid and phosphate metabolism. J Biol Chem 2002, 277:19609-19617.

21. Hoppins S, Collins SR, Cassidy-Stone A, Hummel E, Devay RM, Lackner LL, Westermann B, Schuldiner M, Weissman JS, Nunnari J: A mitochondrial-focused genetic interaction map reveals a scaffold-like complex required for inner membrane organization in mitochondria. J Cell Biol 2011, 195:323-340.

22. Schuldiner M, Collins SR, Thompson NJ, Denic V, Bhamidipati A, Punna T, Inmels J, Andrews B, Boone C, Greenblatt JF, Weissman JS, Krogan NJ: Exploration of the function and organization of the yeast early secretory pathway through an epistatic miniarray profile. Cell 2005, 123:507-519.

23. Costanzo M, Baryshnikova A, Bellay J, Kim Y, Spear ED, Sevier CS, Ding H, Koh JL, Toufighi K, Mostafavi S, Prinz J, St Onge RP, VanderSluis B, Makhnevych T, Vizeacoumar FJ, Alizadeh S, Bahr S, Brost RL, Chen Y, Cokol M, Deshpande R, Li Z, Lin ZY, Liang W, Marback M, Paw J, San Luis BJ, Shuteriqi E, Tong AH, van Dyk N, et al: The genetic landscape of a cell. Science 2010, 327:425-431.

\section{doi:10.1186/1476-0711-13-18}

Cite this article as: Serhan et al:: The polyene antifungals, amphotericin $\mathrm{B}$ and nystatin, cause cell death in Saccharomyces cerevisiae by a distinct mechanism to amphibian-derived antimicrobial peptides. Annals of Clinical Microbiology and Antimicrobials 2014 13:18. 